\title{
Hepatic and renal features in sepsis: etiological, clinical and laboratory correlations
}

\begin{abstract}
Hepatorenal syndrome (HRS) occurring in sepsis has become an accepted approach since 2007, type I HRS being especially characteristic to alterations occurring in sepsis. This study aimed to find associations between elements pertaining to the microbiological, clinical and biochemical aspects in patients with hepatic and/or renal dysfunctions and HRS in sepsis. A total of 117 patients with sepsis were included in the study, patients that had been admitted in the Infectious Disease Clinical Hospital in Iasi, Romania, across a period of 17months, from November 2012 to April 2014, presenting with or developing hepatic or renal functional disorders, HRS, and other organ dysfunctions. We have carried out a statistical analysis of paraclinical parameters in the context of clinical modifications.

Thus, we have identified a series of statistical correlations between the levels of serum creatinine and the degree of severity in sepsis, and also between total bilirubin level and altered neurological status (coma, confusional state). Using the ROC curve analysis, the optimal cut-off values for ALT, AST and total bilirubin were set at $78 \mathrm{IU} / \mathrm{L}, 75.5 \mathrm{IU} / \mathrm{L}$, and $25.5 \mathrm{mg} \%$, respectively, while the Quick Index showed a cutoff value of $66 \%$, in an inversely proportion relationship with unfavourable evolution of liver disease.
\end{abstract}

Keywords: sepsis, hepatic and renal impairment, statistical analysis
Volume 7 Issue 3 - 2017

\author{
Codrina Bejan,' Egidia Miftode,' Lucian \\ Boiculese, ${ }^{2}$ Carmen Dorobat ${ }^{3}$ \\ 'Discipline of Infectious Diseases, Grigore T. Popa University of \\ Medicine and Pharmacy, Romania \\ ${ }^{2}$ Statistics and Bioinformatics Department, Grigore T. Popa \\ University of Medicine and Pharmacy, Romania \\ ${ }^{3}$ Infectious Diseases Hospital of lasi Romania, Grigore T. Popa \\ University of Medicine and Pharmacy, Romania
}

Correspondence: Codrina Bejan, Grigore T. Popa University of Medicine and Pharmacy, lasi, Romania, Tel +407272162 I5, Email bejancodrina@yahoo.com

Received: September 18, 2017 | Published: September 26, 2017

\section{Introduction}

During the 19th century, two authors, Frerichs (1861) and Flint (1863) pointed out an association between advanced impairment of liver function, the presence of ascitic liquid and renal insufficiency with oliguria, without any renal histological changes. ${ }^{1}$ Almost one hundred years later a physiopathology in HRS was described by Hecker and Sherlock.

A main feature of HRS is failure of the renal system, usually, not associated to considerable injuries in kidney anatomy or histology, as the etiopathogenic substrate of HRS rests in a significant vasoconstriction of renal arterial system. ${ }^{2,3}$

A consensus on the definition of HRS was published in 1996 by the International Ascites Club, describing this syndrome as renal failure that occurs in the presence of severe liver disease, acute or chronic, however without the underlying renal pathology. At that time, HRS was an exclusion diagnosis accepted in the absence of hypovolemia, nephrotoxic drugs, sepsis and glomerulonephritis. ${ }^{4}$

Numerous pertinent studies showed the relation between HRS and the renine-angiotensin-aldosteron system (RAAS), sympathetic nervous system (SNS) and renal prostaglandins (PG). ${ }^{5}$ Another vasoconstrictor present in high concentration in HRS is endothelin, although its pathogenic role is not fully understood. ${ }^{5}$ The path physiological characteristic of HRS is intense renal vasoconstriction, multiple mechanisms being involved in the disturbance of systemic hemodynamics, vasoconstrictor systems activation and reduction of vasodilation. ${ }^{3,5}$

A number of hypotheses have been forwarded to explain liver function involvement in HRS and cirrhosis, the most widely accepted ones pointing to arterial vasodilatation and hepatorenal reflex.
Splanchnic arteriolar vasodilatation appears in patients with cirrhosis and portal hypertension mediated most probably by nitric oxide. ${ }^{6}$

Until 2006, HRS definitions had referred to a reversible damage of renal function occurring in patients with advanced liver cirrhosis or fulminant liver failure and is marked by decrease in glomerular filtration rate and renal plasma flow, in the absence of other causes of renal dysfunction. ${ }^{7}$ With the advancement of knowledge, it was proven that the original definition lacked concision regarding numerous exclusion criteria, among them sepsis. ${ }^{8}$ Before revision in 2007, guidelines proposed to exclude bacterial infections from HRS diagnosis. $^{2}$

The triggering factor for HRS is the decreasing in renal blood flow due to vasoconstriction under the influence of multiple neurohumoral factors, activation of rennin-angiotensin-aldosterone system, endothelins and natriuretic peptides. ${ }^{2}$

The HRS process consists of an extreme renal vasoconstriction caused by determined by $\mathrm{Na}+$ retention and vasoconstrictor systems, the result being a very low glomerular filtration rate. ${ }^{10}$ Peripheral arterial vasodilatation theory sustains that splanchnic vasodilatation is a result of portal hypertension from cirrhosis. ${ }^{2}$

Using the available data on the type of HRS, a number of studies indicated that terlipressin improves kidney function by more than $65 \%$ in patients with type $1 \mathrm{HRS}$, significantly increasing survival rate. ${ }^{11-13}$ Patients with sepsis were excluded from all these studies, at that time in line with the initial definitions of HRS. ${ }^{14}$ More recently, Rodriguez E et al. delivered the results of a study assessing the role of terlipressin and albumin in patients with type I HRS and sepsis, revealing a similarity with the results found by studies which had excluded patients with sepsis. ${ }^{14}$ 
HRS has been described as a deficit in the prerenal segment, a preischemic status determined by tubular necrosis due to ischemia, when reduced blood flow results from acute tubular necrosis. ${ }^{15}$ Type 1 HRS is characterized by a rapidly deterioration of renal function and failure. The values of the initial serum creatinine exceeding 2.mg/ $\mathrm{dl}$ or $220 \mu \mathrm{mol} / \mathrm{l}$ in less than 2 weeks'time are suggestive for type I of HRS. ${ }^{17}$

Although type 1 HRS often unfolds with a cascading event, particularly associated with spontaneous bacterial peritonitis. Type 1 HRS occurs within an acute decline in the circulatory function (arterial hypotension and activation of the endogenous vasoconstrictor systems), commonly associated with a rapid worsening of the liver function and encephalopathy, and carries a very poor prospects. ${ }^{17}$ At the moment is accepted that type I mechanism could illustrate the pathophysiology in sepsis.

Type 2 HRS is described by moderate renal failure (serum creatinine level $>1.5 \mathrm{mg} / \mathrm{dl}$ or $133 \mu \mathrm{mol} / \mathrm{l}$ ) following a steady or delayed course, and it is frequently combined with refractory ascites. Survival in patients with type 2 HRS is shorter than in those with ascites but without renal failure. ${ }^{16,17}$

\section{Materials and methods}

The study was run in the Infectious Disease Clinical Hospital, in Iasi, Romania and centred on patients diagnosed with sepsis during the period comprised between November 2012 and April 2014, assessing data both from their observation records and the annexed documents, with a signed informed consent referring to data used for research purposes. We included a number of 117 patients with sepsis associating hepatic/renal dysfunction, hepatorenal syndrome and other organ functional disorders.

For these patients we evaluated the following: demographic data (age, gender, environment of origin), length of hospital stay, disease etiology, initial and succeeding laboratory data, APACHE and CARMELI prognostic scores, the presence of liver and/ or kidney disorders, and the evolution towards recovery or death. Sepsis diagnosis inclusion was in accordance with the definition of suspected or confirmed infection, supported by the systemic inflammatory response syndrome (meeting at least 2 of the following criteria: fever/ hypothermia, tachycardia, tachypnea, and leucocytosis/leukopenia).

Sepsis diagnosis was set in patients with acute organic dysfunction (cardiac, hepatic, renal, respiratory, circulatory failure, disseminated intravascular coagulation or shock). The bacteriology laboratory carried out an analysis of the biological products by direct examination, observation of growth cultures, complement-fixation and latex agglutination tests.

\section{Statistical analysis}

We created an EXCEL database which included descriptive, qualitative and quantitative parameters, while for data processing we used the SPSS 16.0 version, the value $p<0.05$ being considered statistically significant. We applied the $t$ student test, chi-square test, Box Plot charts, the Pearson correlation for normally distributed variables, the Spearman correlation independently of the distribution type and in accordance with the order of rank values, and the Pairwise Comparisons test for data comparison with small samples. Where data was not normally distributed we employed the Mann-Whitney test to confront two sets of variables, while the Kruskal-Wallis test was used for comparing complex data sets. The ROC curve helped us to determine the degree of sensitivity/specificity of diagnostic tests in hepatorenal dysfunction in sepsis.

\section{Results and discussion}

Patients' average age was 62.9years, the median being 65.5. Environment of origin was urban and rural in relatively equal proportions.

Etiological agents in our patients with sepsis were Gram positive germs (39 stems) as: Staphylococcus aureus Meti-S and Meti-R, Staphylococcus epidermidis, Streptococcus spp., Enterococcus spp., as well as Gram negative germs (28 stems): non fermentative Gram negative bacilli, Escherichia coli ESBL, Klebsiella pneumoniae, Proteus mirabilis, Pseudomonas aeruginosa.

There is currently no specific diagnostic test for a clear-cut HRS diagnosis, only a diagnosis of exclusion based on the revised criteria of the International Ascites Club. Primary investigations consisted in determining the glomerular filtration rate, with creatinine clearance $<40 \mathrm{ml} / \mathrm{min}$ or elevated serum creatinine levels $>135 \mu$ moles $/ \mathrm{L}$ once all other causes of renal dysfunction were eliminated. A relevant indicator of the functional capacity encloses natriuresis level $<10 \mathrm{mmol} / \mathrm{L}$, urinary osmolarity $>$ plasma osmolarity, natremia $<130 \mathrm{mmol} / \mathrm{L}$ and diuresis $<500 \mathrm{ml} / 24 \mathrm{hrs}$.

Patients' inclusion criteria referred to sepsis, which, besides the multiple organ dysfunctions, associated hepatic and renal dysfunction. The absence shock previous to setting the diagnosis of hepatorenal syndrome was considered the selection criterion according to the International Ascites Club which defines the association of hepatorenal syndrome in sepsis. ${ }^{17}$

Only $40 \%$ of all patients with sepsis selected for our study developed hepatorenal syndrome. Clearance of serum creatinine in patients with hepatorenal syndrome contrasted to those who did not presented the syndrome associated, calculated by the MDRD equation, showed a median of $26 \mathrm{~mL} / \mathrm{min} / \mathrm{m}^{2}$ (Figure 1).

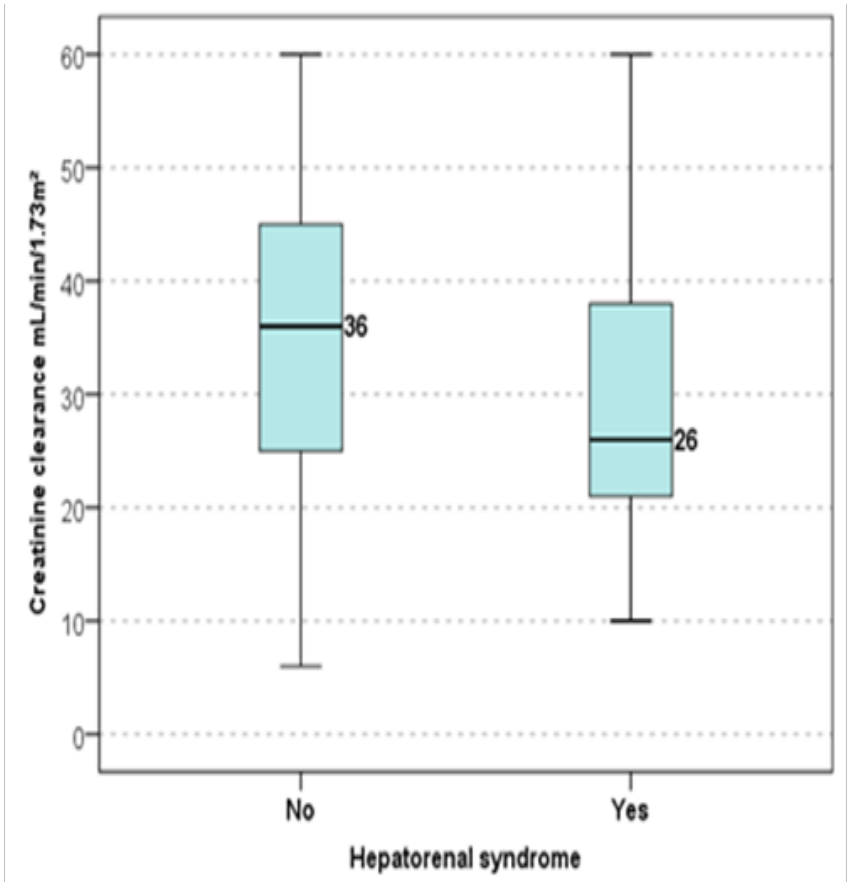

Figure I Serum creatinine in patients with HRS, calculated by the MDRD equation. 
In calculating the APACHE score, values situated between 0-20 (class I), 20-30 (II) and over 30 (III) were considered as reference points. We established statistical correlations between creatinine clearance levels as well as three categories of values to calculate the APACHE score by comparing data sets using the Kruskal Wallis nonparametric test (corresponding to ANOVA) for independent samples, obtaining a significance $p$ value of 0.44 . By comparing pairs, we found a statistical significance only were creatinine level upon admission was concerned for APACHE I compared to APACHE III, and, as shown in Figure 2, the APACHE III group had the lowest creatinine value

The utility of paraclinical tests in highlighting liver dysfunction in hepatorenal syndrome was assessed using sensitivity/specificity curves (Figure 3A-3D). According to the results of statistical computing, the optimal cut-off values in patients with hepatorenal dysfunction in sepsis were $78 \mathrm{IU} / \mathrm{L}, 75.5 \mathrm{IU} / \mathrm{L}$ and $25.5 \mathrm{mg} \%$, for ALT, AST and total bilirubin, respectively, and an inversely proportional Quick Index value of $66 \%$.

We have also tried also to determine statistical correlations between total bilirubin levels and altered neurological status (coma, confusional state), obtaining data that indicated a statistical significance $\mathrm{p}=0.01$. Thus, we found correlations between total bilirubin levels and altered neurological status (coma, confusional state) in patients with hepatorenal dysfunction (Figure 4).

A

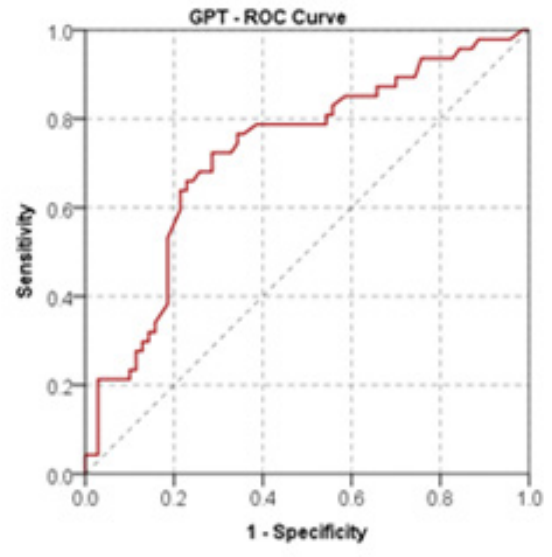

Daponal wymeets are produced by ties.

C

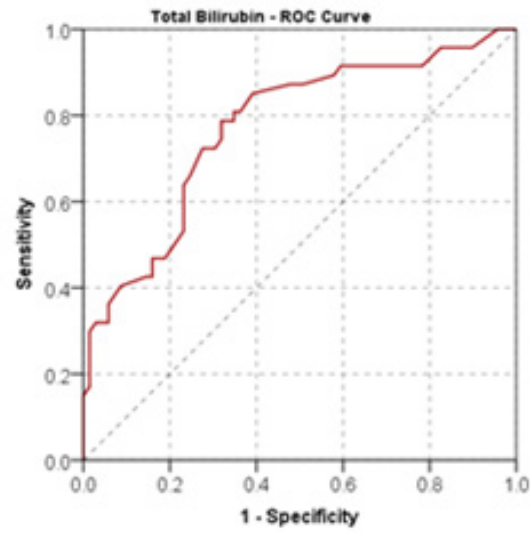

Dingoul wyments are probuced by ties

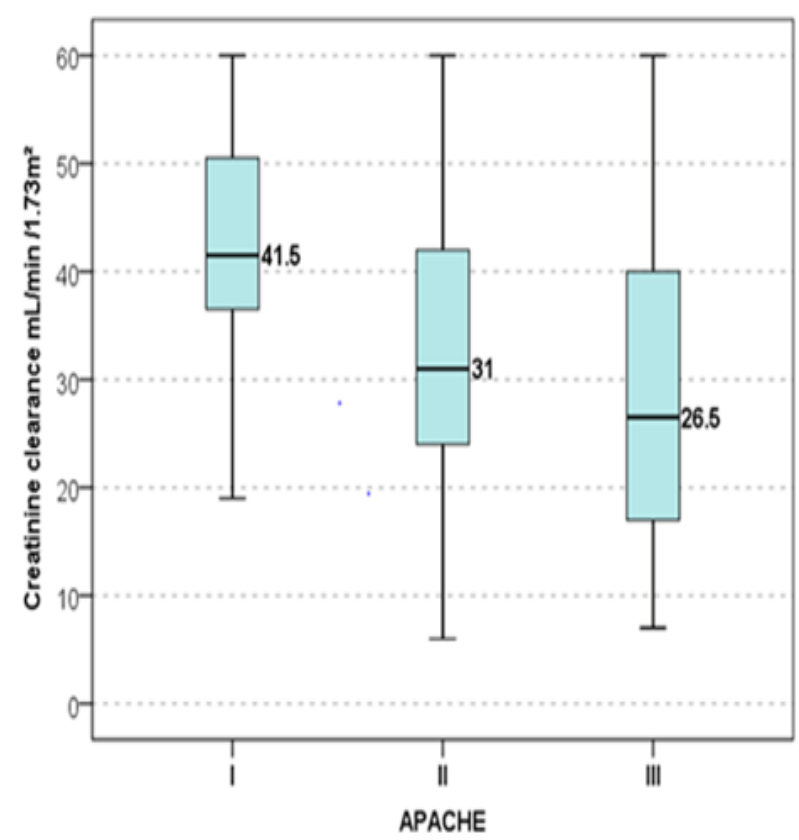

Figure 2 Statistical correlation between serum creatinine clearance and severity degree in sepsis.

B

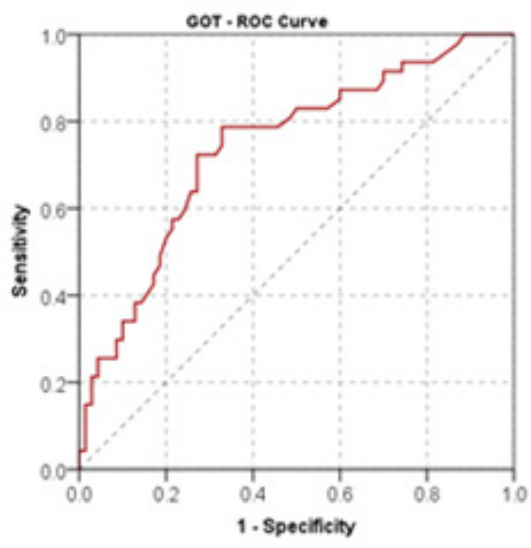

Dapenal regments are produced by tien

D

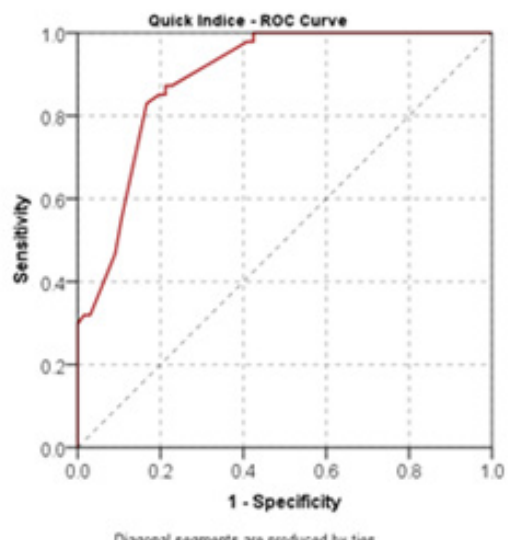

Figure 3 Cut-off curves in hepatorenal dysfunction in sepsis. 


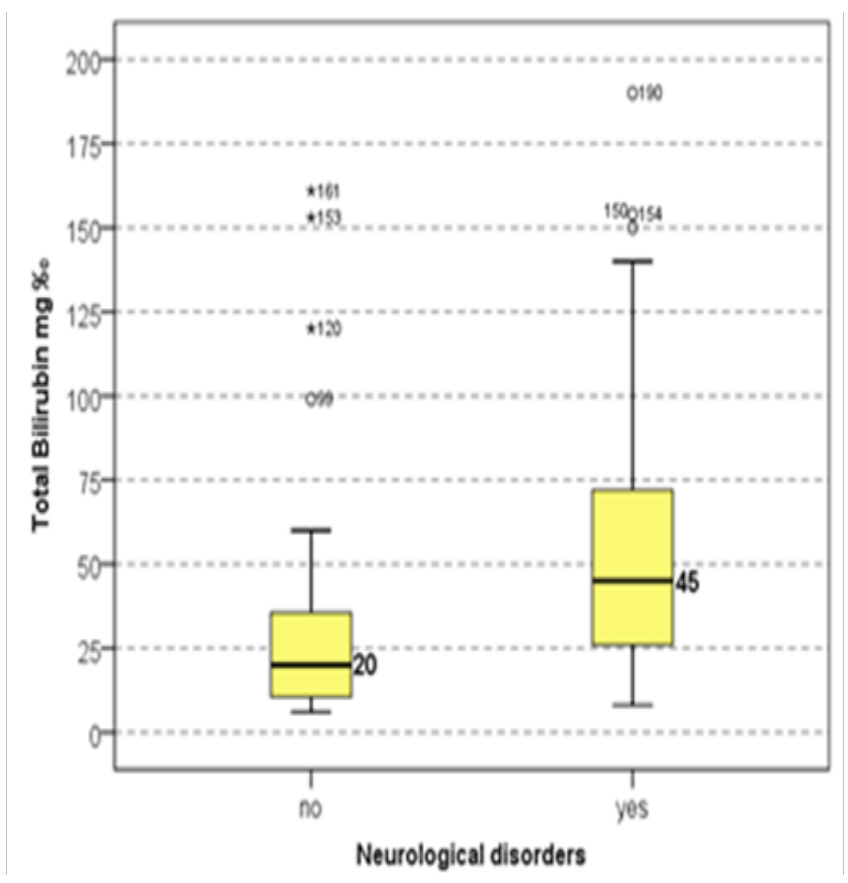

Figure 4 Correlation between total bilirubin and altered neurological status (comma, confusional state).

\section{Conclusion}

In patients with hepatorenal dysfunction within sepsis, we have noticed statistical correlations between the level of serum creatinine and severity of sepsis, as well as between the level of total bilirubin and altered neurological status (coma, confusional state).

Cut-off values on the sensitivity/specificity curve in patients with hepatorenal dysfunction within sepsis were 78IU/L for ALT, 75.5IU/L for AST, $25.5 \mathrm{mg} \%$ o for total bilirubin, while QI had a cut-off value of $66 \%$, in an inversely proportional relationship with unfavourable evolution of liver disease.

Although the standard definition of hepatorenal syndrome applies to precise values of biochemical parameters associated with altered hepatic and renal function, establishing a hepatorenal syndrome diagnosis in critical patients with sepsis remains a challenge for the clinician, as the elements of the clinical and biological evolution picture prove difficult to fit into correlation charts.

\section{Acknowledgements}

We would especially like to thank the Department of Statistics and Bioinformatics of "Grigore T. Popa" University of Medicine and Pharmacy who helped us with data processing.

\section{Conflict of interest}

The author declares no conflict of interest.

\section{References}

1. Flint A. Clinical report on hydro-peritoneum, based on analysis of fortysix cases. Am J Med Sci. 1863;45(90):306-339.

2. Salerno F, Gerbes A, Gines P, et al. Diagnosis, prevention, and treatment of hepatorenal syndrome in cirrhosis. Gut. 2007;56(9):1310-1318.

3. Devuni D. Hepatorenal Syndrome. Medscape; 2016.

4. Enescu A, Petrescu F, Mitruț P, et al. Hepatorenal Syndrome: Diagnosis and Treatment - newsreel. Rom. J Intern Med. 2016;54(3):143-150.

5. Wong F, Moore K, Dingemanse J, et al. Lack of renal improvement with nonselective endothelin antagonism with tezosentan in type 2 hepatorenal syndrome. Hepatology. 2008;47(1):160-168.

6. Al-Khafaji A, Nadim MK, Kellum JA. Hepatorenal disorders. Chest. 2015;148(2):550-558.

7. Wadei HM, Mai ML, Ahsan N, et al. Hepatorenal syndrome: pathophysiology and management. Clin J Am Soc Nephrol. 2006;1:10661079.

8. Arroyo V, Gines P, Gerbes AL, et al. Definition and diagnostic criteria of refractory ascites and hepatorenal syndrome in cirrhosis. International Ascites Club. Hepatology. 1996;23(1):164-176.

9. Arroyo V, Terra C, Gines P. Advances in the pathogenesis and treatment of type-1 and type-2 hepatorenal syndrome. J Hepatol. 2007;46(5):935946.

10. Gines P, Schrier RW. Renal failure in cirrhosis. $N$ Engl $\mathrm{J}$ Med. 2009;361(13):1279-1290.

11. Moreau R, Durand F, Poynard T, et al. Terlipressin in patients with cirrhosis and type 1 hepato-renal syndrome: a retrospective multicenter study. Gastroenterology. 2002;122(4):923-930.

12. Gluud LL, Christensen K, Christensen E, et al. Systematic review of randomized trials on vasoconstrictor drugs for hepatorenal syndrome. Hepatology. 2010;51(2):576-584.

13. Sanyal AJ, Boyer T, Garcia Tsao G, et al. A randomized, prospective, double blind, placebo-controlled trial of terlipressin for type 1 hepatorenal syndrome. Gastroenterology. 2008;134(5):1360-1368.

14. Francoz C, Durand F. Type-1 hepatorenal syndrome in patients with cirrhosis and infection vs. sepsis-induced acute kidney injury: What matters? Journal of Hepatology. 2014;60(5):907-909.

15. Mareau R, Lebrec D. Review article: hepatorenal syndrome- definitions and diagnosis. Aliment Pharmacol Ther. 2004;20:24-28.

16. Kopacova M. Hepatorenal syndrome. World J. Gastroenterol. 2012;18(36):4978-4984.

17. International Club of Ascites Guidelines. 